Tropical Journal of Pharmaceutical Research May 2016; 15 (5): 1053-1060

ISSN: $1596-5996$ (print); 1596-9827 (electronic)

(C) Pharmacotherapy Group, Faculty of Pharmacy, University of Benin, Benin City, 300001 Nigeria.

All rights reserved.

Available online at http://www.tjpr.org

Original Research Article

http://dx.doi.org/10.4314/tjpr.v15i5.22

\title{
Clinical Studies on conformal radiotherapy combined with epidermal growth factor receptor-tyrosine kinase inhibitor in second-line treatment of non-small cell lung cancer
}

\author{
Yong Cai ${ }^{1}$, Ji-Ying Wang ${ }^{2}$ and Chong Bai $^{3 *}$ \\ ${ }^{1}$ Department of Radiation Oncology, ${ }^{2}$ Department of Oncology, Shanghai Pulmonary Hospital, Tongji University School of \\ Medicine, ${ }^{3}$ Department of Respiration, Changhai Hospital, The Second Military Medical University, Shanghai 200433, China \\ *For correspondence: Email: chongbaismmu@126.com; Tel: +86 21 81873232; Fax: +86 2181873232
}

Received: 19 September 2015

Revised accepted: 3 March 2016

\begin{abstract}
Purpose: To study the effect of conformal radiotherapy combined with epidermal growth factor receptor-tyrosine kinase inhibitor (EGFR-TKI) in the second-line treatment of non-small cell lung cancer (NSCLC).

Methods: A total of 316 patients attending Shanghai Pulmonary Hospital affiliated to Tongji University, were divided into two groups: 106 patients were treated with conformal radiotherapy combined with EGFR-TKI (gefitinib, $250 \mathrm{mg} /$ day; or erlotinib, $150 \mathrm{mg} /$ day), while 210 patients were treated with EGFR$T K I$ alone. Some factors, including adverse reactions (AR), disease control rate (DCR), progression-free survival (PFS), overall survival (OS), and one-year and two-year survival rate, were evaluated.

Results: No obvious difference was observed in $A R$ between the two groups $(p>0.05)$. In the combination therapy group, complete response (CR) was 5 cases, partial response (PR) 43 cases, and stable disease (SD) 47 cases, progressive disease (PD) was 11 cases, response rate (RR) was $45.3 \%$, and DCR $89.6 \%$. Median PFS in the combination therapy group and targeted therapy group was 6.5 and 5.0 months, respectively. On the other hand, median OS in the combination therapy group and targeted group was 14.1 and 12.6 months, respectively. One-year survival rate of the combination therapy group and EGFR-TKI group was 60.3 and $50.0 \%$, respectively, while the two-year survival rate was 26.3 and $19.0 \%$, respectively.

Conclusion: Conformal radiotherapy combined with EGFR-TKI can be used as an effective second-line treatment for NSCLC.
\end{abstract}

Keywords: Non-small cell lung cancer, Epidermal growth factor receptor-tyrosine kinase inhibitor, Conformal radiotherapy, Targeted therapy, Survival rate

\begin{abstract}
Tropical Journal of Pharmaceutical Research is indexed by Science Citation Index (SciSearch), Scopus, International Pharmaceutical Abstract, Chemical Abstracts, Embase, Index Copernicus, EBSCO, African Index Medicus, JournalSeek, Journal Citation Reports/Science Edition, Directory of Open Access Journals (DOAJ), African Journal Online, Bioline International, Open-J-Gate and Pharmacy Abstracts
\end{abstract}

\section{INTRODUCTION}

Lung cancer is the primary cause of cancer deaths. In the last 30 years, the incidence of lung cancer in China has increased significantly, especially in females [1,2]. Non-small cell lung cancer (NSCLC) accounts for $80-85 \%$ of lung cancers [3]. Clinically, more than two-thirds of NSCLC patients do not opt for radical surgery when definite diagnosis is made [4]. Currently, radio chemotherapy is the first choice of conventional treatment; however it is limited by poor efficacy and side effects [5-8]. Recently, epidermal growth factor receptor inhibitor (EGFR-TKI), a targeted therapy, has become a hotspot for treating NSCLC. Epidermal growth factor receptor (EGFR) is type I transmembrane tyrosine kinase receptor, a member of ErbB/HER 
family. Studies have found that EGFR inhibitors can enhance the radiosensitivity of cancer cells $[9,10]$.

Radiotherapy is the most powerful local treatment method besides surgery, while targeted therapy has an efficacy close to or even superior to chemotherapy, and the side effects are much fewer than chemotherapy. Currently, combination therapy has become a hot strategy for treating NSCLC. The role of EGFR-TKI in second-line treatment of NSCLC has generally been accepted, but the efficacy and influencing factors of radiotherapy combined with TKI as second-line treatment need further studies. This study investigates the clinical outcomes of conformal radiotherapy combined with EGFR-TKI (combination therapy) in the management of NLCSC vis a vis treatment with EGFR-TKI alone.

\section{EXPERIMENTAL}

\section{Subjects}

NSCLC patients attending Shanghai Pulmonary Hospital affiliated to Tongji University and on second- and third-line treatments from January 2009, were divided into two groups. One group received EGFR-TKI combined with thoracic radiotherapy, while the second group received EGFR-TKI monotherapy. The patients were followed up for a period of 44 months. A total of 316 cases entered the step of study analysis, and clinical data were collected from the patients at Shanghai Pulmonary Hospital affiliated to Tongji University.

\section{Inclusion criteria}

1. All the patients were diagnosed as NSCLS with definite pathological types; in addition, date study commenced, and pathological specimens (including surgical pathological tissues and sections, lung puncture or biopsy specimens, sputum basal cell detection, lymph node or other metastasis puncture or biopsy specimens, fiber optic bronchoscope biopsy or brush biopsy, or lavage fluid basal cell detection, etc).

2. All the patients were diagnosed as NSCLS in stage III or IV, or had treatment failure with firstor second-line treatment (181 patients received EGFR-TKI therapy after first-line treatment failed, and 135 patients received EGFR-TKI therapy after second-line treatment failed.), and did not receive thoracic radiotherapy and EGFR-TKI therapy before study analysis.

\section{Ethics statement}

The experimental protocols were approved by the Institutional Ethics Committee of Shanghai Pulmonary Hospital, Tongji University (approval no. K09-173), and all the patients enrolled were required to read and sign an informed consent form voluntarily before enrollment.

\section{Study design}

The subjects were divided randomly into two groups: 106 patients were treated with conformal radiotherapy combined with EGFR-TKI (gefitinib, $250 \mathrm{mg} /$ day or erlotinib, $150 \mathrm{mg} /$ day), tagged combination therapy group), while 210 patients were treated with EGFR-TKI alone (gefitinib, 250 $\mathrm{mg} /$ day or erlotinib, $150 \mathrm{mg} /$ day), i.e., EGFR-TKI monotherapy group.

Thoracic radiotherapy protocol of the combination therapy group: spiral CT scanning was firstly performed (the scanning ranged from thoracic inlet to costophrenic angle, and layer thickness was $0.5 \mathrm{~cm}$ ). The results were transmitted to the TPS planning system via internet. Thereafter, the target region was sketched and lymph nodes (lymph nodes $>1 \mathrm{~cm}$ ) and lung primary tumor were enlarged and seen at the target regions (including mediastinum and hilus pulmonis). Based on the images, the gross tumor volume (GTV) and planning target volume (PTV) were sketched out, expanded PTV by 0.5 $\sim 1.5 \mathrm{~cm}$ according to individual patient's conditions, own breathing exercises, lesion location, position error and other factors, and then sketched out the basic structure of the threatened organs (commonly heart, esophagus, spinal cord, bilateral lung tissues, etc). Normal lung tissues were evaluated with the percentage of 20 Gy radiation volumes in the total lung volume (V20). All the combination therapy patients received conventional-dose conformal radiotherapy, $90 \%$ of total PTV: $40.35 \sim 64.27$ Gy, $1.8 \sim 2$ Gy/day, 5 times / week (Table 1).

Table 1: Operating conditions for thoracic conformal radiotherapy

\begin{tabular}{|c|c|c|c|}
\hline Variable & $\mathbf{N}$ & Variable & $\mathbf{N}$ \\
\hline Dose & 106 & $\begin{array}{l}\text { Palliative } \\
\text { radiotherapy }\end{array}$ & 58 \\
\hline 40 50 Gy & 18 & $\begin{array}{l}\text { Radical } \\
\text { radiotherapy }\end{array}$ & 48 \\
\hline $\begin{array}{l}\text { 50 60 Gy } \\
>60 \text { Gy }\end{array}$ & $\begin{array}{l}61 \\
27\end{array}$ & $\begin{array}{c}\text { Lung V20 } \\
\leq 25 \% \\
>25 \%\end{array}$ & $\begin{array}{l}93 \\
13\end{array}$ \\
\hline
\end{tabular}

ADx-ARMS EGFR gene mutation detection kit (21 kinds) and ADx-ARMS KRAS gene mutation detection kit (7 kinds) (Amoy Diagnostics Co., 
Ltd) were used detect EGFR and KRAS mutations respectively.

\section{Efficacy evaluation criteria used}

Short-term efficacy evaluation was based on chest computed tomography (CT) after 3 months of treatment. Response evaluation criteria for solid tumors (RECIST) were used for the evaluation. Efficacy included: complete response (CR), partial response (PR), stable disease (SD) and progressive disease (PD). Response rate $(R R): C R+P R / t o t a l$ number of cases. Disease control rate $(D C R): C R+P R+S D /$ total number of cases.

\section{Evaluation of long-term efficacy}

It included progression-free survival (PFS, time from start of treatment to tumor progression, last follow-up visit or death), the median overall survival (OS), and one-year and two-year survival rate.

\section{Evaluation of adverse reactions}

Table 1: Demographic characteristics and clinical profile of the patients

\begin{tabular}{|c|c|c|c|c|}
\hline Parameters & $\begin{array}{c}\text { Combination } \\
\text { therapy group } \\
(n=106)\end{array}$ & $\begin{array}{c}\text { EGFR-TKI mono- } \\
\text { therapy group } \\
(n=210)\end{array}$ & $x^{2}$ & $P$-value \\
\hline Age & & & 1.204 & 0.273 \\
\hline$\leq 70$ & 27 & 66 & & \\
\hline$>70$ & 79 & 144 & & \\
\hline Gender & & & 1.562 & 0.211 \\
\hline Male & 61 & 136 & & \\
\hline Female & 45 & 74 & & \\
\hline ECOG score & & & 0.904 & 0.636 \\
\hline 0 & 11 & 22 & & \\
\hline 1 & 67 & 122 & & \\
\hline 2 & 28 & 66 & & \\
\hline Smoking history & & & 1.076 & 0.300 \\
\hline$>300$ year cigarettes & 51 & 114 & & \\
\hline$\leq 300$ year cigarettes & 55 & 96 & & \\
\hline \multicolumn{5}{|l|}{ Position of primary lesion } \\
\hline Upper lobe & 24 & 58 & & \\
\hline Median lobe and lower lobe & 82 & 152 & 0.908 & 0.341 \\
\hline \multicolumn{5}{|l|}{ TNM staging } \\
\hline IIIA & 23 & 59 & & \\
\hline IIIB & 40 & 89 & & \\
\hline IV & 43 & 62 & & \\
\hline Pathological type & & & 4.068 & 0.131 \\
\hline Squamous carcinoma & 30 & 57 & & \\
\hline Adenocarcinoma & 55 & 118 & & \\
\hline Adenosquamous carcinoma & 8 & 13 & & \\
\hline Large cell carcinoma & 9 & 15 & 0.675 & 0.954 \\
\hline Unspecified types & 4 & 7 & & \\
\hline
\end{tabular}

Drug reactions were evaluated according to the WHO anti-cancer drug side effects indexing standards, and radiotherapy reactions were evaluated according to the USA RTOG (Radiation Therapy Oncology Group) acute radiation injury grading standards.

\section{Statistical analysis}

SPSS 13.0 software was used for Chi-squared test for univariate analysis, while Kaplan-Meier method was used to calculate survival rate. Verification was carried out using Logrank method. Logistic regression was used to analyze interstitial pneumonia-related factors, and Cox regression model was used for survival multivariate analysis. Statistical significance level was set at $p<0.05$.

\section{RESULTS}

A total of 316 entered the study, including 197 males and 119 females, aged 32 - 79 years, with a median age of 63 years old (Table 1). 
and the median lung V20 was $21 \%$ (9 - $29 \%$ ). The length of esophagus receiving 45 Gy and 55 Gy radiation were $8.1 \mathrm{~cm}(0 \sim 17.2 \mathrm{~cm})$ and 4.7 $\mathrm{cm}(0-15.3 \mathrm{~cm})$, respectively.

The results of the texts done on the specimens showed that EGFR mutation rate in adenocarcinoma was about $45.2 \%$, the total EGFR mutation rate was about $30.1 \%$, KRAS mutation rate was about $10.1 \%$, and only three patients developed both EGFR and KRAS mutations (Table 3).

In our present study, no obvious difference was observed for the adverse reaction (AR) between the two groups $(p>0.05)$ (Table 4). For interstitial pneumonia, logistic regression analysis showed that smoking and V20 were related to the occurrence of interstitial pneumonia (Table 5).

Table 3: Gene test results

\begin{tabular}{lc}
\hline Gene test & $\mathbf{N}$ \\
\hline EGFR mutation & 95 \\
KRAS mutation & 32 \\
Double mutations & 3 \\
Double negative & 186 \\
\hline
\end{tabular}

Table 4: Adverse reactions in the two groups (N, \%)
In the combination therapy group, CR was in 5 cases, PR in 43 cases, SD in 47 cases, PD in 11 cases, RR $45.3 \%$, and DCR $89.6 \%$; in the targeted therapy group, CR was in 7 cases, PR in 45 cases, SD in 84 cases, PD in 74 cases, RR $24.8 \%$ and DCR $64.8 \%$, and the differences in $\mathrm{RR}$ and DCR were statistically significant (Table $6)$.

In the combination therapy group, median PFS was 6.5 months and the median OS was 14.1 months; in the targeted therapy group, the median PFS was 5.0 months and the median OS was 12.6 months. In the combination therapy group, the one-year survival rate was $60.3 \%$ and the two-year survival rate was $26.3 \%$; in the targeted therapy group, the one-year survival rate was $50 \%$ and the two-year survival rate was $19 \%$ : PFS $\left(x^{2}=3.706, p=0.054\right)$, OS $\left(x^{2}=\right.$ 3.308, $p=0.069)$ (Fig 1).

In the combination therapy group, KRAS mutation and TNM staging correlated with survival, as risk factors, while EGFR mutation correlated with survival, as a protective factor. In patients with EGFR mutation, the median PFS was 9.7 months and the median OS was 16.3 months; in the patients with KRAS mutation, the median PFS was 2.6 months and the median OS was 6.3 months (Table 7).

\begin{tabular}{lcccc}
\hline \multicolumn{1}{c}{ Adverse reaction } & $\begin{array}{c}\text { Combination } \\
\text { therapy group } \\
(\mathbf{N = 1 0 6 )}\end{array}$ & $\begin{array}{c}\text { EGFR-TKI mono- } \\
\text { therapy group } \\
(\mathbf{N = 2 1 0})\end{array}$ & $\mathbf{X}^{2}$ & $\boldsymbol{P}$-value \\
\hline Rash G1-2 & $55(51.9 \%)$ & $107(60.0 \%)$ & 0.025 & 0.875 \\
Peeling & $16(15.1 \%)$ & $29(13.8 \%)$ & 0.095 & 0.758 \\
Oral ulcer & $11(10.4 \%)$ & $19(9.0 \%)$ & 0.145 & 0.703 \\
Interstitial pneumonia G1-2 & $16(15.1 \%)$ & $21(10 \%)$ & 1.768 & 0.184 \\
Esophagitis G1-3 & $43(40.6 \%)$ & $25(11.9 \%)$ & 34.264 & $<0.001$ \\
Nausea and vomiting & $11(10.4 \%)$ & $19(9.0 \%)$ & 0.145 & 0.703 \\
Diarrhea & $8(7.5 \%)$ & $22(10.5 \%)$ & 0.703 & 0.402 \\
Granulopenia & $12(11.3 \%)$ & $18(8.6 \%)$ & 0.620 & 0.431 \\
Reduced hemachrome & $15(14.2 \%)$ & $23(11.0 \%)$ & 0.681 & 0.409 \\
\hline
\end{tabular}

Table 5: Interstitial pneumonia-related risk factors

\begin{tabular}{lccccc}
\hline Variable & $\mathbf{B}$ & $\mathbf{X}^{2}$ & $\boldsymbol{P}$-value & OR & 95 \% Cl \\
\hline Smoking & 0.254 & 4.119 & 0.0424 & 1.29 & $1.02-3.21$ \\
V20 & 0.168 & 7.291 & 0.0069 & 1.18 & $1.07-2.29$ \\
\hline
\end{tabular}

Table 6: Short-term efficacy in the two groups

\begin{tabular}{lcccc}
\hline Variable & $\begin{array}{c}\text { Second-line } \\
\text { combination group }\end{array}$ & $\begin{array}{c}\text { Second-line } \\
\text { targeting group }\end{array}$ & $\mathbf{X}^{2}$ & $\boldsymbol{P}$-value \\
\hline RR & $45.3 \%$ & $24.8 \%$ & 13.714 & 0.000 \\
DCR & $89.6 \%$ & $64.8 \%$ & 22.142 & 0.000 \\
\hline
\end{tabular}


Table 7: Prognosis multivariate analysis by Cox method for combination therapy

\begin{tabular}{cccccc}
\hline Variable & $\boldsymbol{\beta}$ & $\mathbf{X}^{2}$ & $\mathbf{p}$ & $\mathbf{H R}$ & $\mathbf{9 5} \mathbf{C l}$ \\
\hline KRAS & 0.288 & 6.70 & 0.030 & 1.33 & $1.21-3.36$ \\
TNM staging & 0.453 & 18.00 & 0.000 & 1.54 & $1.34-5.52$ \\
EGFR & -0.403 & 4.87 & 0.0027 & 0.67 & $0.33-0.85$ \\
\hline
\end{tabular}

A Survival Functions

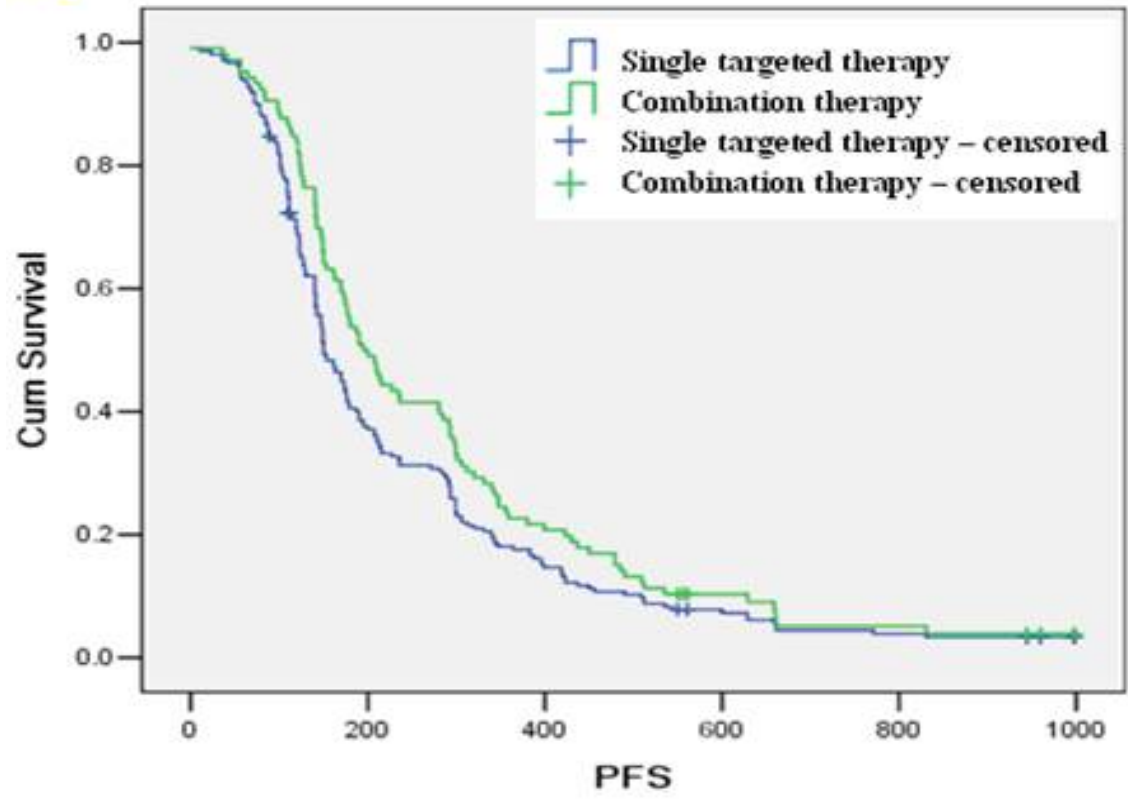

B

Survival Functions

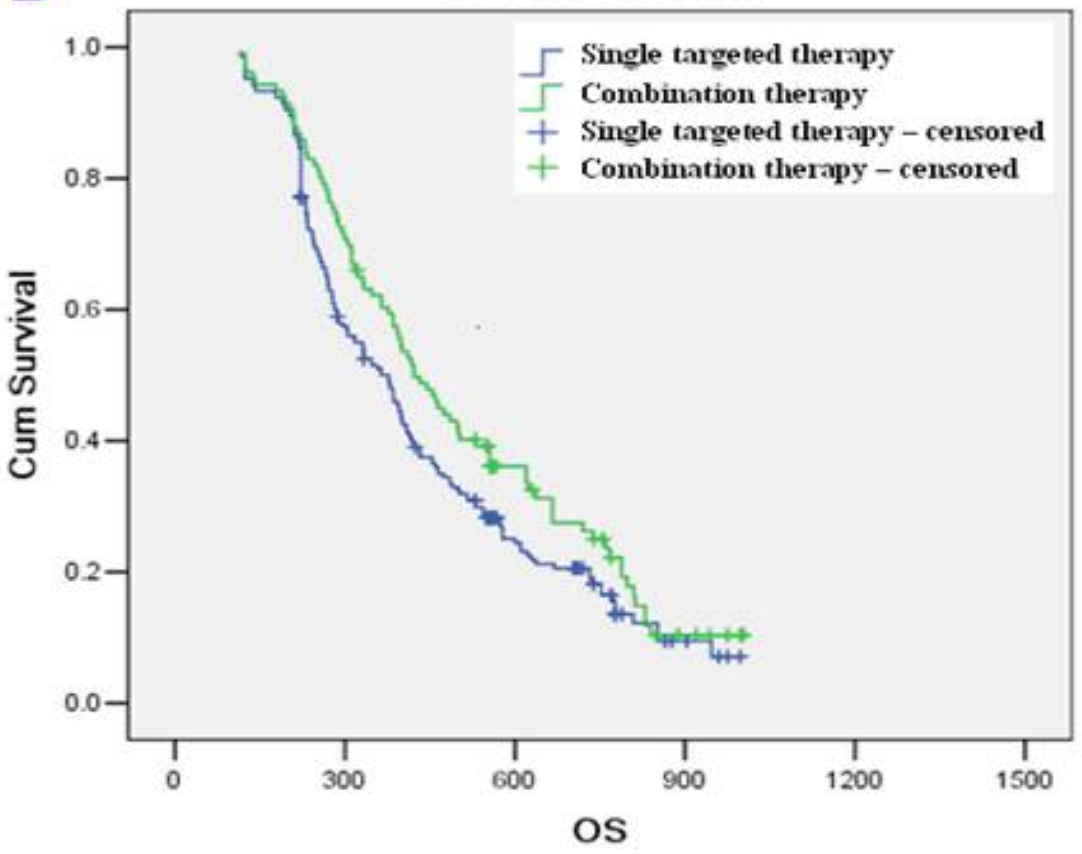

Fig 1: PFS and OS curves for the two patient groups

\section{DISCUSSION}

Thoracic radiotherapy has been considered the most effective method for the treatment of primary tumor besides surgery. Conformal radiotherapy has become a standard radiotherapy for lung radiotherapy [13-15]. Additionally, radiotherapy can significantly reduce side effects of radiotherapy. However, radiotherapy is a topical treatment method. The major lung cancer has been in locally advanced when detected clinically. For these patients in 
middle and advanced stages, the efficacy of radiotherapy is limited, and it must be combined with drugs to achieve the treatment goals [1115].

Molecular targeted therapy, as the greatest development in NSCLC treatment method, has been widely used clinically, and EGFR-TKI has become a hotspot in this field. The effects of EGFR-TKI as a basic drug in second-line treatment of NSCLC have obtained a consensus, and the National Comprehensive Cancer Network (NCCN) has also recommended EGFRTKI as a first-line drug for patients with EGFR mutations [16]. Meanwhile, the efficacy of synchronous radiotherapy and chemotherapy in treatment of NSCLC is superior to that of sequential radiotherapy and chemotherapy [17]. However, the side effects of synchronous treatment have increased significantly. Compared with chemotherapy drugs, EGFR-TKI has significantly less side effects and can even be used for patients with PS $>2$ points. The most obvious adverse reaction of thoracic radiotherapy is radiation pneumonitis. Interstitial pneumonia could be induced by both the radioactive rays and EGFR-TKI. Thus, for synchronous treatment, lung is the common target of lethal side effects, and the incidence of interstitial pneumonia increases significantly. In clinical trials CALGB 30106 and SWOG 9504, EGFR-TKI was combined with thoracic radiotherapy, and serious lung toxic reactions did not occur in the radiation field, suggesting that EGFR-TKI combined with thoracic radiotherapy is safe and feasible $[18,19]$. As the two most common methods for EGFR and KRAS gene detection, direct DNA sequencing method and real-time PCR technology-based ARMS method have their own characteristics. The sequencing method is currently the gold standard for detection and can detect known and unknown EGFR mutations, and ARMS method can detect small amounts of cells and has higher sensitivity. Our experiment mainly aimed at sensitive EGFR mutations and determination of short and long-term efficacy of radiotherapy, sensitive mutations were known mutations concentrated on nos. 19 and 21 loci, and the mutation types were known, so we chose the high-sensitivity ARMS method for detection.

The results of the present study showed that as a main prognostic factor for EGFR efficacy evaluation, the detection rate of EGFR mutations during the whole treatment was very low, and only less than $10 \%$ of the patients underwent genetic testing for EGFR. This might be due to high financial cost of such test in the study site and also due to low level of medical insurance by the patients which might be related to the testing expense of more than two thousand yuan and no medical insurance.

By testing the pathological specimens collected by the pathology department, this investigation found that EGFR mutation rate for adenocarcinoma (45.2 \%) was significantly higher than other types of NSCL. The EGFR mutation rate in female, non-smoking and adenocarcinoma patients was $82.7 \%$ KRAS mutation rate was about $10.1 \%$, far below the mutation rate of nearly $30 \%$ earlier reported in western countries, supporting the fact that the EGFR mutation rate in eastern countries was high, while the KRAS mutation rate in western countries was high [20,21]. The KRAS mutation rate in patients with smoking $>300$ year cigarettes reached $16.4 \%$, suggesting that KRAS mutations are correlated with smoking. Only 3 cases developed both EGFR and KRAS mutations, indicating that EGFR genes and KRAS genes are mutually exclusive.

Our results revealed that proportion of males was higher than females by nearly $25 \%$, and nearly $30 \%$ of the patients with ECOG PS score $=2$ points received two and three-line treatment, suggesting that after recurrence or metastasis after chemotherapy, the patients' PS score decreased significantly. In all cases, the cases in IV stage accounted for $33.2 \%$, and the rest were cases in IIIA and IIIB stages, suggesting that after chemotherapy failed, the patients' stages increased significantly. In all the cases, the proportion of adenocarcinoma was significantly higher than other types of NSCLC. In first and second-line chemotherapy, the protocol was generally changed after 2 - 4 cycles of chemotherapy failed. The two groups received thoracic radiotherapy combined with EGFR-TKI therapy and EGFR-TKI monotherapy respectively, and the differences in age, gender, ECOG score, stages, pathological types and EGFR and KRAS mutations between the two groups were not statistically different.

All cases received EGFR-TKI therapy after progression, including erlotinib in 163 cases and gefitinib in 153 cases, and the selection of targeting drugs was not specific. 106 patients received EGFR-TKI therapy combined with thoracic radiotherapy. Only 48 patients received radical radiotherapy due to early stages, and 58 patients received palliative radiotherapy. NCCN did not recommend thoracic radiotherapy for stage IV patients, so the number of stage IV cases receiving thoracic radiotherapy was limited, but many studies found that thoracic radiotherapy for stage IV patients could improve local efficacy and long-term survival to a certain 
extent. We chose V20 as the main evaluation indicator for lung radiotherapy reactions and found that the patients with V20 below $25 \%$ accounted for $87.7 \%$, and the requirements in these plans made the incidence of radiation pneumonitis within an acceptable range.

After treatment, the hematologic toxicity, gastrointestinal reactions, rash and mucosa reactions in the two groups were almost similar; however incidence of esophagitis and interstitial pneumonia in combination therapy group increased, which were not seen in the targeted therapy group, and except esophagitis. Lungs were the common target point of radiotherapy and EGFR-TKI therapy, and results showed that the key points for lower incidence of interstitial pneumonia in the combination therapy group were lung V20 and smoking status, and in the patients with V20 less than $20 \%$ and smoking index lower than 300 year cigarettes, the incidence of radiation pneumonitis rate fell below $10 \%$, and only grade 1 radiation pneumonitis occurred, suggesting that no smoking or less smoking and $\mathrm{V} 20<20 \%$ is the safety margin for combination therapy.

In the combination therapy group, RR was 45.3 $\%$ and DCR was $89.6 \%$, significantly higher than $24.8 \%$ and $64.8 \%$ in the targeted therapy group, and the differences were statistically significant, showing the advantages of radiotherapy in local treatment. So far, in addition to surgery, radiotherapy is still the most effective local treatment method. When combined with EGFRTKI therapy, radiotherapy plays a synergistic treatment role and is significantly superior to short-term efficacy of targeted therapy.

The median PFS in combination therapy group was 6.5 months, 1.5 months more than that in targeted therapy group; the median OS in combination therapy group was 14.1 months, 1.5 months more than that in targeted therapy group. No obvious differences in PFS and OS was observed between the two groups, however, when combined with thoracic radiotherapy, PFS and OS were slightly increased in the combination therapy group. Previous investigation suggested that synchronous chemotherapy combined with erlotinib maintenance therapy is an effective and well tolerated method in treatment of unresectable stage III NSCLC. All patients in the reported study were Caucasians, smoking and complicated by squamous carcinoma, but the median erlotinib maintenance still reached 18.7 months [22]. In our study, the median OS in the combination therapy group was 14.1 months, appearing as promising survival due to many of them were in stage IV.

In the cases receiving EGFR-TKI therapy combined with thoracic radiotherapy, EGFR mutations, KRAS mutations and tumor stages were associated with survival, EGFR was a radiation-sensitizing factor, the short-term efficacy on EGFR mutations reached $70-80 \%$, and PFS of patients with EGFR mutations was more than that in patients without mutations. In cases of EGFR mutations, the median PFS was 9.7 months and the median OS was 16.3 months, and PFS and OS of patients with EGFR mutations were more than those of patients receiving combination therapy. Mutations of KRAS genes encoding EGFR downstream GTPase was the main reason for drug resistance in NSCLC patients, and in the cases receiving combination therapy, patients with KRAS mutations had the median PFS of 2.6 months and the median OS of 6.3 months. PFS and OS were significantly less than those in the whole combination therapy group, suggesting that KRAS mutation was the reason for EGFR-TKI resistance and also a factor for radiotherapy resistance.

\section{Limitations of the study}

Not all the patients in the targeted therapy group treated with EGFR-TKI were selected by gene determination. Therefore, some of the patients were not sensitized to TKI treatment, which will be considered in a further study in the future.

\section{CONCLUSION}

Collectively, the findings of this study demonstrate that conformal radiotherapy combined with EGFR-TKI can be used as an effective strategy in the second-line treatment of NSCLC.

\section{CONFLICT OF INTEREST}

No conflict of interest associated with this work.

\section{CONTRIBUTION OF AUTHORS}

We declare that this work was done by the authors named in this article and all liabilities pertaining to claims relating to the content of this article will be borne by the authors. 


\section{REFERENCES}

1. Jemal A, Bray F, Center MM, Ferlay J, Ward E, Forman D. Global cancer statistics. CA Cancer J Clin, 2011; 61: 69-90.

2. Li SG, Zhang XT, Zhang LP. Analyze the risk factors of female lung cancer. Chin J Lab Diagn, 2011; 15: 17671769.

3. Peng $W, W u$ JG, Jiang YB, Liu YJ, Sun T, Wu N, Wu CJ. Antitumor activity of 4-O-(2"-O-acetyl-6"-O-p-coumaroyl$\beta$-D-glucopyranosyl)-p-coumaric acid against lung cancers via mitochondrial-mediated apoptosis. Chem Biol Interact, 2015; 233: 8-13

4. Siegel R, Ma JM, Zou ZH, Jemal A, Jemal A. Cancer statistics, CA Cancer J. Clin. 64, 2014; 2014: 9-29.

5. Zhou DA, Wang LH, Fu XL. The New Comprehensive Therapeutics of Lung Cancer. Shanghai: Fudan University Press, 2009: 81-89.

6. Liao ZX, Jiang GL. Modern Therapeutics of Tumor Radio. Shanghai: Shanghai Science and Technology Press, 2003: 496-497.

7. Greenlee R, Murray T, Bolden S, Wingo PA. . Cancer statistics. CA Cancer J Clin, 2000; 50: 7-33.

8. Pass HI, Mitchell JB, Johnson DH. Molecular Genetics, the Etiology of Lung Cancer and the Epidemiology. Beijing: People's Medical Publishing House, 2002: 81 353.

9. Zhang XJ, Qiang L, Song LH. New developments of targeted therapy in lung cancer. Chinese Journal of Cancer Prevention and Treatment, 2010; 17(7): 551 554.

10. Colquhoun AJ, Mchugh LA, Tulchinsky E, Kriajevska M, Mellon JK. Combination treatment with ionising radiation and gefitinib (Iressa, ZD1839), an epidermal growth factor receptor (EGFR) inhibitor, significantly inhibits bladder cancer cell growth in vitro and in vivo. $J$ Radiat Res (Tokyo), 2007; 48 (5): 351-360.

11. Miyata $H$, Sasaki $T$, Kuwahara $K$, et al. The effects of ZD1839 (Iressa), a highly selective EGFR tyrosine kinase inhibitor, as a radiosensitiser in bile duct carcinoma cell lines. Int J Oncol, 2006; 28: 915-921.

12. Steene JV, Linthout N, Mey J, Vinh-Hung V, Claassens $C$. Definition of gross tumor volume in lung cancer: interobserver variability. Radiother Oncol, 2002; 62: 3749.

13. Caldwell CB, Math $K$, Ung YC, et al. Observer variation in contouring gross tumor volume in patients with poorly defined non-small cell lung tumor on CT: the impact of 18FDG-hybrid PET fusion. Int $J$ Radiat Oncol Biol Phys, 2001; 51: 923-931.
14. Magnani P, Carretta A, Rizzo G, Fazio F, Vanzulli $A$. FDG/PET and spiral CT image fusion for mediastina lymph node assessment of non-small cell lung cancer patients. J Cardivasc Surg, 1999; 40: 741-748

15. Giraud $P$, Antoine M, Larrouy A, Milleron B, Callard $P, D e$ Rycke $Y$, Carette MF, Rosenwald JC, Cosset JM, Housset M. Evaluation of microscopic tumor extension in non-small-cell lung cancer for three-dimensional conformal radiotherapy planning. Int J Radio Oncol Biol Phys, 2000; 48: 1015-1024.

16. Li D, Zhang L, Zhou J, Chen H. Cigarette smoke extract exposure induces EGFR-TKI resistance in EGFRmutated NSCLC via mediating Src activation and EMT. Lung Can, 2016; 93:35-42.

17. Bhardwaj B, Revannasiddaiah S, Bhardwaj H, Balusu S, Shwaiki A. Molecular targeted therapy to improve radiotherapeutic outcomes for non-small cell lung carcinoma. Ann Transl Med, 2016; 4: 50.

18. Ready N, Janne PA, Bogart J, Dipetrillo T, Garst J, Graziano S, Gu L, Wang X, Green MR, Vokes EE. Chemoradiotherapy and gefitinib in stage III Non-small Cell Lung Cancer with epidermal growth factor receptor and KRAS mutation analysis: cancer and leukemia group B (CALEB) 30106, a CALGB-stratified phase II trial. J Thorac Oncol, 2010; 5: 1382-1390.

19. Gandara DR, Chansky K, Albain KS, Leigh BR, Gaspar $L E$, Lara $P N \mathrm{Jr}$, Burris $H$, Gumerlock $P$, Kuebler JP. Consolidation docetaxel after concurrent chemoradiotherapy in stage IIIB non-small cell lung cancer: Phase III southwest oncology group study S9504. J Clin Oncol, 2003; 21(10): 2004-2010.

20. Chen YM, Lai CH, Chang HC, Chao TY, Tseng CC, Fang WF, Wang CC, Chung $Y H$, Wang $Y H$, Su MC. The impact of clinical parameters on progression-free survival of non-small cell lung cancer patients harboring EGFR-mutations receiving first-line EGFR-tyrosine kinase inhibitors. Lung Can, 2016; 93: 47-54.

21. Zhang Q, Sun T, Kang P, Qian K, Deng B, Zhou J, Wang $R$, Jiang $B$, Li K, LiU $F$. Combined analysis of rearrangement of $A L K, R O S 1$, somatic mutation of EGFR, KRAS, BRAF, PIK3CA, and mRNA expression of ERCC1, TYMS, RRM1, TUBB3, EGFR in patients with non-small cell lung cancer and their clinical significance. Cancer Chemother Pharmacol 2016; DOI: 10.1007/s00280-016-2969-y.

22. Casal J, Villanueva MJ, Sergio V, Barón-Duarte FJ, Alonso-Jáudenes G, Santomé L, Afonso-Afonso FJ, Amenedo M, Huidobro G, Campos-Balea B. Eerlotinib as maintenance therapy after concurrent chemoradiotherapy in patients with stage-III non-small cell lung cancer: A Galician Lung Cancer Group phase II study. J Thorac Oncol, 2009; 4: 3-9. 